\title{
Near-Inertial Oscillations of Geophysical Surface Frontal Currents
}

\author{
ANGELO Rubino \\ Institut für Meereskunde, Universität Hamburg, Hamburg, Germany \\ SERGEY DOTSENKO \\ Marine Hydrophysical Institute, Sevastopol, Ukraine \\ PETER BRANDT \\ Institut für Meereskunde an der Universität Kiel, Kiel, Germany
}

(Manuscript received 26 February 2002, in final form 27 January 2003)

\section{ABSTRACT}

\begin{abstract}
Intrinsic oscillations of stable geophysical surface frontal currents of the unsteady, nonlinear, reduced-gravity shallow-water equations on an $f$ plane are investigated analytically and numerically. For frictional (Rayleigh) currents characterized by linear horizontal velocity components and parabolic cross sections, the primitive equations are reduced to a set of coupled nonlinear ordinary differential equations. In the inviscid case, two periodic analytical solutions of the nonlinear problem describing 1) the inertially reversing horizontal displacement of a surface frontal current having a fixed parabolic cross section and 2) the cross-front pulsation of a coastal current emerging from a motionless surface frontal layer are presented. In a linear and in a weakly nonlinear context, analytical expressions for field oscillations and their frequency shift relative to the inertial frequency are presented. For the fully nonlinear problem, solutions referring to a surface frontal coastal current are obtained analytically and numerically. These solutions show that the currents oscillate always superinertially, the frequency and the amplitude of their oscillations depending on the magnitude of the initial disturbance and on the squared current Rossby number. In a linear framework, it is shown that disturbances superimposed on the surface frontal current are standing waves within the bounded region, the frequencies of which are inertial/ superinertial for the first mode/higher modes. In the same frame, a zeroth mode, which could be interpreted as the superposition of an inertial wave on a background vorticity field, would formally yield subinertial frequencies. For surface frontal currents affected by Rayleigh friction, it is shown that the magnitude of the mean current decays according to a power law and that the oscillations decay faster, because this decay follows an exponential law. Implications of the intrinsic oscillations and of their rapid dissipation for the near-inertial motion in an active ambient ocean are discussed.
\end{abstract}

\section{Introduction}

Almost one-half of the energy contained in the oceanic internal-wave band belongs to near-inertial waves (Munk 1981). Because these disturbances, mostly generated in the upper ocean by the action of the wind, can transfer a consistent part of their energy downward, they represent one of the major sources of energy available for the production of mixing and turbulence in the interior ocean (e.g., Kunze 1985; Balmforth et al. 1998; Garrett 2001). In this energy transmission from the nearsurface layers to the abyss, equatorward propagation and interaction with oceanic mesoscale features play fundamental roles (e.g., Kunze 1985, 1986; Wang 1991;

Corresponding author address: Angelo Rubino, Institut für Meereskunde, Universität Hamburg, Troplowitzstr. 7, D-22529 Hamburg, Germany.

E-mail: rubino@ifm.uni-hamburg.de
Klein and Treguier 1993; Lee and Eriksen 1997; Young and Jelloul 1997; Chant 2001; Garrett 2001). On the $f$ plane, the transformation of oceanic near-inertial disturbances having similar spatial scales as the generating atmospheric fronts into smaller-scale near-inertial waves, which are able to propagate their energy downward rapidly, is attributed to the interactions of these long near-inertial waves with background inhomogeneities in the flow field such as those associated with mesoscale fronts and vortices (e.g., Kunze and Sanford 1984; Kunze 1985; Mied et al. 1986; Wang 1991; Zervakis and Levine 1995; Lee and Eriksen 1997; van Meurs 1998; Chant 2001). Near-inertial internal waves are in fact affected by variations in the background relative vorticity, buoyancy, and divergence: variations in the background fluid relative vorticity are able to induce variations in the lower bound of the internal-wave frequency band, because they force variations in the wave 
vector and modulations in the wave amplitude (Mooers 1975; Kunze 1985); variations in the horizontal density structure experienced by near-inertial internal waves during their evolution can produce variations in the upper bound of their wave band, because they affect the density contrast experienced by water particles along their orbits (Lee and Eriksen 1997); and background divergent or convergent flows can cause wave decay or growth (Kunze 1985; Rubenstein and Roberts 1986; Lee and Eriksen 1997).

On the other hand, the same coherent mesoscale frontal features involved in the transformation of the spatial scales of oceanic near-inertial internal waves are known to possess intrinsic near-inertial modes of oscillations. For instance, circular nonlinear frontal (warm core) vortices of the reduced-gravity shallow-water equations characterized by different structures of their velocity and thickness fields pulsate inertially: shoaling and expansions, deepenings and contractions, alternate during an exact inertial period (Cushman-Roisin 1987; Rogers 1989; Rubino et al. 1998). The robustness of these oscillations has been recently demonstrated in the frame of a theoretical study carried out by Rubino et al. (2002) for nonlinear geophysical circular frontal vortices evolving on an $f$ plane under the influence of harmonic horizontal eddy viscosity, linear and quadratic interfacial friction, and linear water entrainment; Rubino and Brandt (2003) reported, in the frame of an experimental investigation carried out using the large-scale turntable of the Laboratory of Geophysical and Industrial Fluid Flows (LEGI) (Grenoble, France), that the same oscillations emerge for similar mesoscale frontal features generated by the impulsive release of a motionless cylindrical body of lighter water on an $f$ plane. Elliptical nonlinear frontal (warm core) vortices of the reduced-gravity shallow-water equations having parabolic sections and velocity components linearly dependent on the horizontal coordinates show a more complex, intriguing oscillatory behavior: subinertial, superinertial, and inertial oscillations can in fact coexist (Young 1986; Cushman-Roisin 1987). Thus, if, on the one hand, these mesoscale features influence the preexisting, wind-generated large-scale near-inertial wave field, on the other hand, they act, according to their intrinsic modes of oscillations, as generators of smaller-scale near-inertial waves when, as a response to larger-scale disturbances, they are forced to oscillate in a stratified ocean. Extending our knowledge on possible intrinsic oscillations inherent in oceanic mesoscale frontal features may contribute to a better understanding of the intricate dynamic interactions leading to the observed near-inertial wave field in the ocean. As a condition, a deeper comprehension of the intrinsic oscillations of such frontal features in a simplified context may be important.

In this investigation, we present analytical and numerical results that describe aspects of the near-inertial, inviscid and frictional intrinsic oscillations of stable geophysical surface frontal currents of special shapes

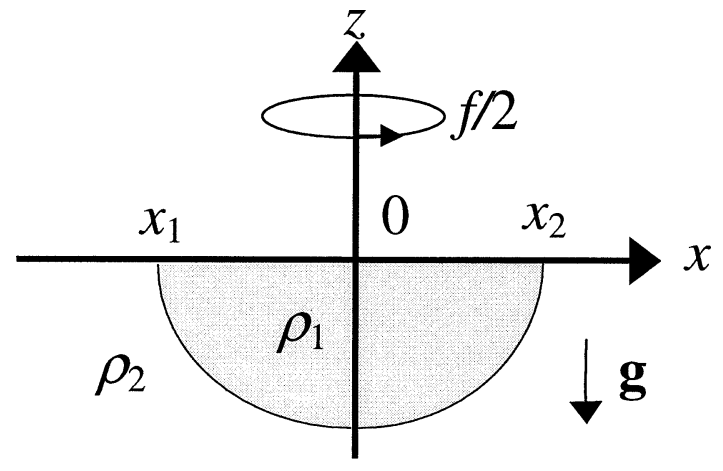

FIG. 1. Schematic representation of a surface frontal current in the framework of the reduced-gravity theory. The current is bounded by the outcropping lines located at $x=x_{1}$ and $x=x_{2}$.

and velocity structures in the framework of the nonlinear, reduced-gravity shallow-water equations.

The paper is organized as follows. In section 2, the reduction of the nonlinear, reduced-gravity model to a system of ordinary differential equations (ODEs) for special horizontal structures is performed. Two exact analytical solutions are presented in section 3 . In section 4 , linear and weakly nonlinear solutions for the inviscid case are presented and the attenuation of a coastal current and its near-inertial oscillations caused by linear friction is investigated. In section 5, the frequency of disturbances superimposed on the surface frontal current is analyzed in a linear context and the results are compared with the results of Kunze (1985). In section 6, the results of the study are summarized and a discussion is presented.

\section{The governing equations}

The plane motion of a surface frontal layer (Fig. 1) on an $f$ plane in the frame of the nonlinear, reducedgravity, frictional shallow-water model is governed, in a nondimensional form, by the equations

$$
\begin{aligned}
\frac{\partial u}{\partial t}+u \frac{\partial u}{\partial x}-v & =-\frac{\partial h}{\partial x}-s u, \\
\frac{\partial v}{\partial t}+u \frac{\partial v}{\partial x}+u & =-s v, \quad \text { and } \\
\frac{\partial h}{\partial t}+\frac{\partial(h u)}{\partial x} & =0
\end{aligned}
$$

where $x$ is the horizontal coordinate, $t$ is the time, and $(u, v)$ and $h$ are the components of the horizontal velocity and the thickness of the surface layer, respectively. The first two equations of (1) include a linear Rayleigh friction with a constant friction coefficient $s$.

The scales for $x, t$, and $s$ are the internal Rossby radius of deformation Ro $=f^{-1} c$, the inertial period $f^{-1}$ (divided by $2 \pi$ ), and the inertial frequency $f$, respectively. The value $c=\sqrt{g^{\prime} h^{+}}$is the phase velocity of the linear internal gravitational long waves, $g^{\prime}=g\left(1-\rho_{1} / \rho_{2}\right)$ is 
the reduced gravity, $g$ is the acceleration due to gravity, $\rho_{1}$ and $\rho_{2}$ are the densities of the fluid within the front and in its surroundings respectively, and $h^{+}$is the maximum surface layer thickness at the initial stage. This thickness is scaled by $h^{+}$, and the horizontal velocity components are scaled by $c$. It is assumed that $h>0$ within the strip $x_{1}<x<x_{2}$, and $h=0$ at $x=x_{1}(t)$ and $x=x_{2}(t)$ (see Fig. 1).

We assume now that the fields are characterized by the following horizontal structure:

$$
\begin{aligned}
& u=a_{0}(t)+a_{1}(t) x, \quad v=b_{0}(t)+b_{1}(t) x, \quad \text { and } \\
& h=c_{0}(t)+c_{1}(t) x+c_{2}(t) x^{2} .
\end{aligned}
$$

Substitution of (2) into (1) yields the following system of seven nonlinear, coupled ODEs in the unknown variables $a_{0}, a_{1}, b_{0}, b_{1}, c_{0}, c_{1}$, and $c_{2}$ :

$$
\begin{aligned}
\dot{a}_{0}+a_{0} a_{1}-b_{0}+c_{1}+s a_{0} & =0, \\
\dot{a}_{1}+a_{1}^{2}-b_{1}+2 c_{2}+s a_{1} & =0, \\
\dot{b}_{0}+a_{0} b_{1}+a_{0}+s b_{0} & =0, \\
\dot{b}_{1}+a_{1} b_{1}+a_{1}+s b_{1} & =0, \\
\dot{c}_{0}+a_{0} c_{1}+a_{1} c_{0} & =0, \\
\dot{c}_{1}+2 a_{0} c_{2}+2 a_{1} c_{1} & =0, \quad \text { and } \\
\dot{c}_{2}+3 a_{1} c_{2} & =0 .
\end{aligned}
$$

Note that the dots indicate temporal derivation. For every time, two conditions constraining the variables that describe the layer thickness have to be met: The first is $c_{2}<0$, required to ensure the existence of two outcropping lines; the second is $\mu=c_{1}^{2}-4 c_{0} c_{2}>0$, required to ensure that $h>0$ for $x_{1}<x<x_{2}$. From (3) we find

$$
\begin{aligned}
\dot{c}_{2} & =-3 a_{1} c_{2} \quad \text { and } \quad \dot{\mu}=-4 a_{1} \mu, \text { i.e., } \\
c_{2}(t) & =c_{2}(0) e^{-3 I(t)} \quad \text { and } \quad \mu(t)=\mu(0) e^{-4 I(t)},
\end{aligned}
$$

where $I=\int_{0}^{t} a_{1}(\tau) d \tau$. Thus, if the surface frontal current has a finite width at $t=0$, it will not expand to infinity or degenerate into a line for $t>0$.

We specify now the velocity field and the surface layer thickness expressed by (2) for the description of a surface frontal coastal current; that is, we consider a lateral wall located at $x=0$ (see Fig. 2). We thus impose $u=0$ along this line, that is, $a_{0} \equiv 0$, and hence

$$
\begin{aligned}
b_{0}-c_{1} & =0, & \dot{a}_{1}+a_{1}^{2}-b_{1}+2 c_{2}+s a_{1} & =0, \\
\dot{b}_{0}+s b_{0} & =0, & \dot{b}_{1}+a_{1} b_{1}+a_{1}+s b_{1} & =0, \\
\dot{c}_{0}+a_{1} c_{0} & =0, & \dot{c}_{1}+2 a_{1} c_{1} & =0,
\end{aligned}
$$

and

$\dot{c}_{2}+3 a_{1} c_{2}=0$.

From the first, the third, and the sixth equation of (4) we find $c_{1}\left(a_{1}-s / 2\right)=0$. There are two possibilities.

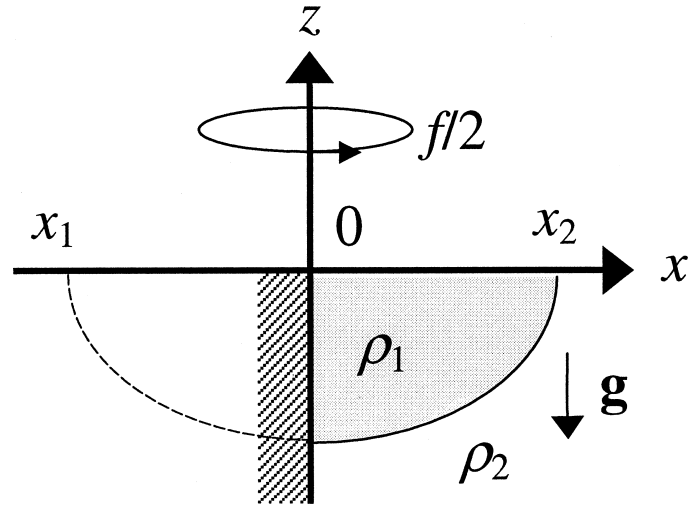

FIG. 2. Schematic representation of a surface frontal coastal current in the framework of the reduced-gravity theory. The current is bounded by the coast at $x=0$ and by the outcropping line at $x=x_{2}$.

In the first case is $a_{1}=s / 2$, and, to avoid contradictions between the equations of (4), it must be $s=0$, which implies $a_{1}=0$. Allowed solutions are thus stationary, geostrophic frictionless currents:

$$
\begin{aligned}
& u=0, \quad v=c_{1}+2 c_{2} x=\partial h / \partial x, \quad \text { and } \\
& h=c_{0}+c_{1} x+c_{2} x^{2} .
\end{aligned}
$$

The second case $\left(c_{1}=0\right)$ implies $b_{0}=0$, which means that the model represented by (1) and (2) is applicable to unsteady surface frontal coastal currents constrained by a straight wall if the fields (2) are written as

$$
\begin{aligned}
& u=A(t) x, \quad v=B(t) x, \quad \text { and } \\
& h=C(t) x^{2}+D(t) .
\end{aligned}
$$

The unknown functions in (5) satisfy the initial-value problem:

$$
\begin{aligned}
\dot{A} & =-s A+B-2 C-A^{2}, \\
\dot{B} & =-A-s B-A B, \\
\dot{C} & =-3 A C, \quad \dot{D}=-A D, \\
A(0) & =A_{0}, \quad B(0)=B_{0}, \quad C(0)=C_{0}, \quad \text { and } \\
D(0) & =D_{0},
\end{aligned}
$$

with $C_{0}<0$ and $D_{0}>0$.

\section{Analytical solutions of surface frontal current oscillations}

In the inviscid case, two exact analytical solutions of the nonlinear models (3) and (6) can be found. The first resembles a solution describing steady rotations of elliptical vortices (Cushman-Roisin et al. 1985; Young 1986); the second is similar to solutions used to describe 1) pulsations of an infinitely long ribbon at the sea bottom (Frei 1993) and 2) barotropic oscillations in a channel of parabolic cross section (Shapiro 1996). In the frame of our investigation, exact analytical solutions of 
(3) and (6) represent oscillations of mesoscale surface frontal features.

\section{a. The motion of a surface frontal current of invariant form}

We search for a solution of (3) describing the inviscid, unsteady dynamics of a surface frontal current of invariant form. The surface frontal layer width and maximum thickness (see Fig. 1) are

$$
x_{2}-x_{1}=-\mu^{1 / 2} c_{2}^{-1} \text { and } h^{+}=-0.25 \mu c_{2}^{-1},
$$

respectively. Because, for a surface frontal current of invariant form, $c_{2}$ must be constant, we can define $c_{2}$ $=-k$, where $k$ is a positive constant. From (3) we find

$$
a_{1}=0 \text { and } b_{1}=-2 k \text {, }
$$

which allows us to replace (3) with the simplified system of ODEs:

$$
\begin{aligned}
\dot{a}_{0}-b_{0}+c_{1} & =0, \quad \dot{b}_{0}-(2 k-1) a_{0}=0, \\
\dot{c}_{1}-2 k a_{0} & =0, \quad \text { and } \quad \dot{c}_{0}+a_{0} c_{1}=0 .
\end{aligned}
$$

Introducing the obtained solution of (8) in (2) yields

$$
\begin{aligned}
& u=A_{0} \sin \Phi, \\
& v=B_{0}-(2 k-1) A_{0} \cos \Phi-2 k x, \text { and } \\
& h=C_{0}-k\left[x-x_{0}(t)\right]^{2},
\end{aligned}
$$

where

$$
\Phi=t+\varphi \quad \text { and } \quad x_{0}=\frac{B_{0}}{2 k}-A_{0} \cos \Phi .
$$

Note that $A_{0}, B_{0}, C_{0}>0, k>0$, and $\varphi$ are arbitrary constants. In (9), all fields oscillate exactly inertially. This behavior differs from that of elliptical vortices with a fixed shape, for which the rotation frequency can be either subinertial or superinertial (Cushman-Roisin et al. 1985; Young 1986). Note that, in both cases, the center of mass oscillates exactly inertially (Ball 1963; Young 1986; Cushman-Roisin 1987).

\section{b. Oscillation of a surface frontal coastal current}

The initial-value problem (6) can be solved analytically (Frei 1993; Shapiro 1996). Consider a surface frontal coastal current emerging from a motionless initial state-that is, $A_{0}=B_{0}=0$, and $C_{0}<0$. In this case, the exact solution of (6) can be written as

$$
\begin{aligned}
t & = \begin{cases}\psi\left(m_{2}, m\right) & \text { if } \dot{m} \geq 0 \quad \text { and } m_{2}<m \leq m_{3}, \\
2 \psi\left(m_{2}, m_{3}\right)-\psi\left(m_{2}, m\right) & \text { if } \dot{m} \leq 0 \quad \text { and } m_{2} \leq m<m_{3},\end{cases} \\
\psi\left(m_{2}, m\right) & =\int_{m_{2}}^{m} \sqrt{\frac{-\xi}{\left(\xi-m_{1}\right)\left(\xi-m_{2}\right)\left(\xi-m_{3}\right)}} d \xi=\frac{2 m_{2}}{\sqrt{m_{3}\left(m_{2}-m_{1}\right)}} \Pi\left(\alpha, 1-\frac{m_{2}}{m_{3}}, \beta\right), \\
\alpha & =\arcsin \sqrt{\frac{\left(m-m_{2}\right) m_{3}}{m\left(m_{3}-m_{2}\right)}}, \quad \text { and } \beta=\sqrt{\frac{m_{1}\left(m_{2}-m_{3}\right)}{\left(m_{2}-m_{1}\right) m_{3}},}
\end{aligned}
$$

where the function $m=m(t)$ is defined as $A=m^{-1} \dot{m}$, where $m=m_{1,2,3}\left(m_{1}<0<m_{2}<m_{3}\right)$ are real roots of the cubic equation $m^{3}+p_{1} m^{2}-p_{2} m+1=0$, with $p_{1}=-2\left(-4 C_{0}\right)^{-1 / 3}$ and $p_{2}=-\left(1+4 C_{0}\right)\left(-4 C_{0}\right)^{-2 / 3}$. The function $\Pi(\alpha, k, \beta)$ in (11) is known as the elliptic integral of the third kind (Gradshteyn and Ryzhik 1980). From (10) it follows that the oscillations characterizing this solution have period $T=2 \psi\left(m_{2}, m_{3}\right)$. The estimation

$$
\begin{aligned}
\psi\left(m_{2}, m_{3}\right) & <\int_{m_{2}}^{m_{3}} \frac{d \xi}{\sqrt{\left(\xi-m_{2}\right)\left(m_{3}-\xi\right)}} \\
& =2 \int_{0}^{1} \frac{d \xi}{\sqrt{1-\xi^{2}}}=\pi
\end{aligned}
$$

implies that a surface frontal coastal current of any amplitude oscillates always superinertially, that is, $T<2 \pi$. In another context (i.e., an infinitely long ribbon at the sea bottom), a similar behavior was found numerically by Frei (1993). Although this result gives a limit for the oscillation frequency, from the solution (10) and (11) the quantification of other characteristics of the surface frontal dynamics is not straightforward. For this reason, in the next section we will quantify these characteristics in the frame of a linear and a weakly nonlinear theory, as well as numerically.

\section{Linear and weakly nonlinear oscillations, and frictional decay of surface frontal coastal currents}

\section{a. Linear and weakly nonlinear oscillations around geostrophy}

We now consider small-amplitude oscillations around geostrophy: $u=0, v=-2 \gamma x=\partial h / \partial x$, and $h=1-$ $\gamma x^{2}$ for $0 \leq x \leq x_{2}$, where $\gamma$ is the squared current 
Rossby number. To this purpose, let us introduce the coefficients $a, b, c$, and $d$ defined by

$$
\begin{aligned}
& A=a(t), \quad B=-2 \gamma+b(t), \\
& C=-\gamma+c(t), \text { and } D=1+d(t) .
\end{aligned}
$$

Substitution of (12) into (6) leads to the following system of ODEs:

$$
\begin{aligned}
& \dot{a}=b-2 c-a^{2}, \quad \dot{b}=(2 \gamma-1) a-a b, \\
& \dot{c}=3 \gamma a-3 a c \text {, and } \dot{d}=-a-a d,
\end{aligned}
$$

with $a(0)=a_{0}, b(0)=b_{0}, c(0)=c_{0}$, and $d(0)=d_{0}$.

\section{1) LINEAR OSCILLATIONS AROUND GEOSTROPHY}

In the linear case, the initial-value problem (13) takes the form

$$
\begin{aligned}
\ddot{a}+\omega^{2} a & =0, \quad a(0)=a_{0}, \quad \text { and } \\
\dot{a}(0) & =b_{0}-2 c_{0} .
\end{aligned}
$$

It represents the harmonic oscillations

$$
a=\left(b_{0}-2 c_{0}\right) \omega^{-1} \sin \omega t+a_{0} \cos \omega t
$$

with superinertial frequency

$$
\omega=\sqrt{1+4 \gamma}
$$

It results also in

$$
\begin{aligned}
& \quad \begin{array}{l}
b=2 c_{1}-(1-2 \gamma) p(t), \quad c=c_{1}+3 \gamma p(t), \\
\text { where } p=-\dot{a} / \omega^{2} \text {, and in }
\end{array} \\
& \qquad \begin{array}{l}
d=d_{1}-p(t), \\
c_{1}=\omega^{-2}\left[3 \gamma b_{0}+(1-2 \gamma) c_{0}\right] \text { and } \\
\qquad d_{1}=\omega^{-2}\left[\omega^{2} d_{0}-\left(b_{0}-2 c_{0}\right)\right] .
\end{array}
\end{aligned}
$$

The conditions $c_{0}<\gamma$ and $d_{0}>-1$ (the satisfaction of which ensures that the thickness of the surface frontal coastal current is positive) and $\max _{t}\{c(t)\}<\gamma$ and $\min _{t}\{d(t)\}>-1$ must be satisfied for any time. These restrictions, in fact, ensure that the current will not expand to infinity or degenerate into a line. The solution (15)-(18) shows that infinitesimal disturbances of surface frontal coastal currents in geostrophic equilibrium yield always superinertial oscillations [see (16)] around this equilibrium.

\section{2) WEAKLY NONLINEAR OSCILLATIONS AROUND GEOSTROPHY EMERGING FROM A CROSS-FRONT DISTURBANCE}

We now consider weakly nonlinear oscillations caused by a disturbance that, initially, is merely cross frontal (i.e., $a_{0} \neq 0, b_{0}=c_{0}=d_{0}=0$ ). From the second and third equation of (13), we find

$$
\begin{aligned}
& b(t)=(1-2 \gamma)[E(t)-1] \text { and } \\
& c(t)=\gamma\left[1-E^{3}(t)\right],
\end{aligned}
$$

where $E=\exp \left[-\int_{0}^{t} a(\xi) d \xi\right]$. This allows replacement of (13) with

$$
\begin{array}{rlrl}
\dot{a} & =\omega^{2} \delta-a^{2}+6 \gamma \delta^{2}+2 \gamma \delta^{3}, & \dot{\delta} & =-a-a \delta, \\
a(0) & =\varepsilon, \text { and } \quad \delta(0)=0,
\end{array}
$$

where $\delta(t)=E(t)-1$ is a new unknown function and $\varepsilon=a_{0}$ is a small parameter.

To find the solution of (20) we apply a perturbation method in $\varepsilon$ (e.g., Nayfen 1981). First we replace the independent variable $t$ by a new variable $\tau$ using the following expansion:

$$
t=\tau\left(1+\alpha_{1} \varepsilon+\alpha_{2} \varepsilon^{2}+\alpha_{3} \varepsilon^{3}+\cdots\right),
$$

which contains the unknown constants $\alpha_{j}$. Note that (21) describes the nonlinear frequency distortion of the frequency of the linear solutions (16). We then write the unknown functions $a(t)$ and $\delta(t)$ as power series in $\varepsilon$ :

$$
a=\sum_{j=1}^{\infty} a_{j}(\tau) \varepsilon^{j} \quad \text { and } \quad \delta=\sum_{j=1}^{\infty} \delta_{j}(\tau) \varepsilon^{j}
$$

Substitution of (21) and (22) into (20) leads to a set of equations in $a_{j}$ and $\delta_{j}$ for the corresponding powers of $\varepsilon$. We have solved analytically this problem for the first three orders:

$$
\begin{aligned}
a= & \varepsilon\left(1+\frac{1+\gamma-67 \gamma^{2}}{4 \omega^{6}} \varepsilon^{2}\right) \cos \Omega t+\varepsilon^{2} \frac{4 \gamma}{\omega^{3}} \sin \Omega t \\
& -\varepsilon^{2} \frac{1+8 \gamma}{2 \omega^{3}}\left(\sin 2 \Omega t-\varepsilon \frac{8 \gamma}{\omega^{3}} \cos 2 \Omega t\right) \\
& -\varepsilon^{3} \frac{1+17 \gamma+61 \gamma^{2}}{4 \omega^{6}} \cos 3 \Omega t+O\left(\varepsilon^{4}\right) \text { and } \\
\Omega= & \omega\left[1-\frac{3 \gamma(1-\gamma)}{\omega^{6}} \varepsilon^{2}\right]^{-1}+O\left(\varepsilon^{4}\right) .
\end{aligned}
$$

Because the periodic oscillations of the frontal coastal current described by (13) are nonsinusoidal, a spectral analysis of its exact solution would reveal the presence of the fundamental oscillation frequency $\Omega$ together with all of its higher harmonics. In the second-order approximation, instead, the front oscillation is composed of a superposition of first and second harmonics only. Every successive order of weakly nonlinear solutions leads to the appearance of an additional harmonic of the fundamental oscillation frequency $\Omega$. Note that, in the range of geophysical coastal currents $(\gamma<$ $1)$, the second-order correction to the linear oscillation frequency results in an increase of the oscillation frequency.

A comparison among asymptotic solution (23), linear solution (15), and exact solution (obtained numerically using a fourth-order Runge-Kutta method; e.g., Abramowitz and Stegun 1972) of (13) is shown in Fig. 3. Note that, although the asymptotic solution (23) is very close to the exact solution, a mismatch between linear 


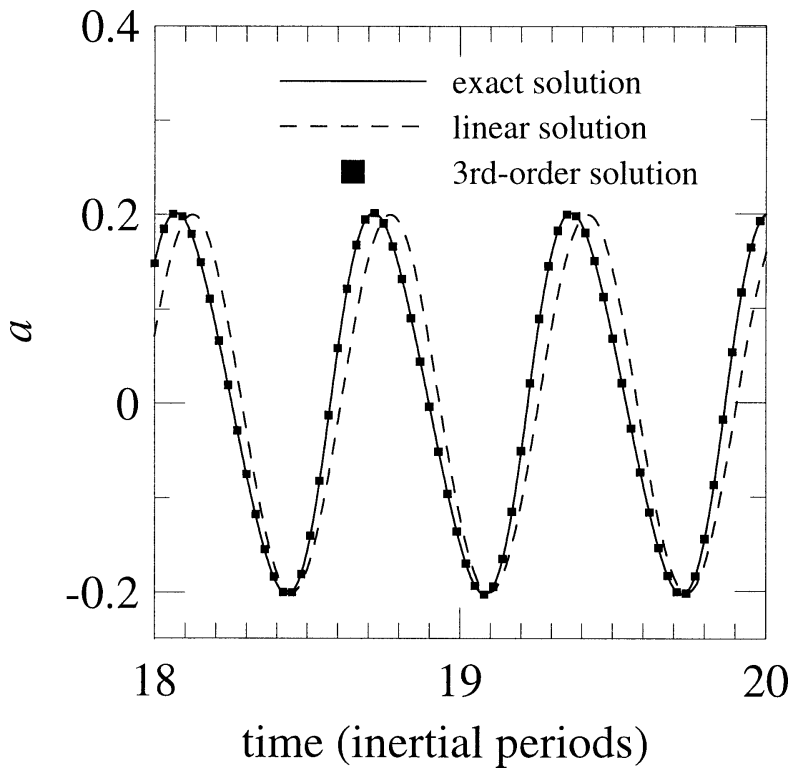

FIG. 3. Temporal evolution of $a(t)$ between 18 and 20 inertial periods for the inviscid case. Presented are different solutions of (13): the exact (numerical) solution, the weakly nonlinear third-order solution (23), and the linear solution (15). The initial values used in (13) are $a_{0}=0.2, b_{0}=c_{0}=d_{0}=0$, and $\gamma=0.347$.

and nonlinear solution is visible and results from the neglect of the amplitude dependence of the oscillation frequency in the linear solution.

\section{3) WEAKLY NONLINEAR OSCILLATIONS AROUND GEOSTROPHY EMERGING FROM AN ALONGFRONT DISTURBANCE}

We now consider nonlinear oscillations caused by initial alongfront disturbances perturbing a surface frontal coastal current in geostrophic equilibrium - that is, we assume that, in (13), $a_{0}=c_{0}=d_{0}=0$ and $b_{0}=\varepsilon$, where $\varepsilon$ is a small parameter. In this case, (20) may be replaced with

$$
\begin{aligned}
& \dot{a}=\omega^{2} \delta+\varepsilon+\varepsilon \delta-a^{2}+6 \gamma \delta^{2}+2 \gamma \delta^{3}, \\
& \dot{\delta}=-a-a \delta, \quad \text { and } a(0)=\delta(0)=0 .
\end{aligned}
$$

Substitution of (21) and (22) into (24) leads to a set of equations in $a_{j}$ and $\delta_{j}$ for the corresponding powers of $\varepsilon$. We have solved analytically this problem for the first three orders and found the expression for the oscillation frequency:

$$
\Omega=\frac{\omega}{1+\alpha_{1} \varepsilon+\alpha_{2} \varepsilon^{2}+O\left(\varepsilon^{3}\right)},
$$

where

$$
\alpha_{1}=\frac{6 \gamma}{\omega^{4}} \quad \text { and } \quad \alpha_{2}=\frac{15 \gamma(-1+3 \gamma)}{\omega^{8}} .
$$

From (25) it can be evinced that the frequency shift due to nonlinearity may be both positive $\left(b_{0}>0\right)$ and neg-

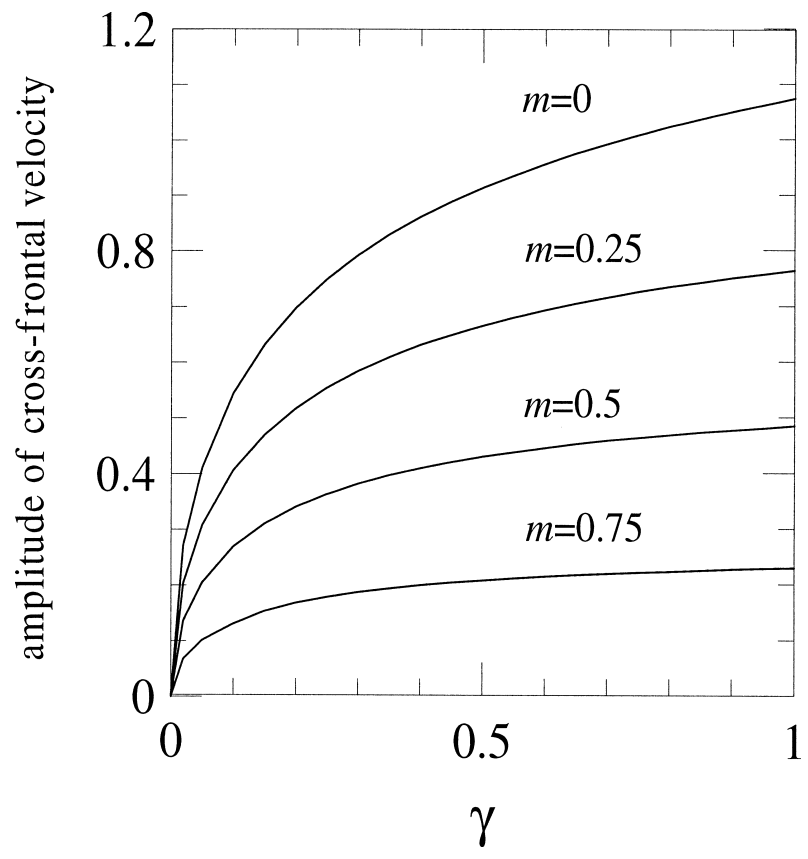

FIG. 4. Amplitude of cross-frontal velocity oscillations as function of the initial squared current Rossby number $\gamma$ for different current deviations from geostrophy. The values of the parameter $m$ vary from 0 to 1 , which corresponds to consideration of currents whose initial structures range from quiescence $(m=0)$ to geostrophy $(m=1)$.

ative $\left(b_{0}<0\right)$. On the contrary, in the case of a mere cross-front initial disturbance, only frequencies that are larger than in the linear case can be obtained, because the frequency shift depends in that case on $\varepsilon^{2}$ only. Thus, in our solutions, nonharmonic oscillations depend on the kind of initial disturbances, and their frequency is always larger than the inertial one.

For currents characterized by different current Rossby numbers, the amplitude of the current oscillations and the deviation of the oscillation periods from the inertial period can be investigated by considering initial deviations from geostrophy. To this purpose, we define $b_{0}$ $=2 \gamma(1-m)$, and we vary the parameter $m$ in the range of $0 \leq m \leq 1$. The value $m=0$ refers to an initially motionless surface frontal layer; for $m=1$, the current is in exact geostrophic equilibrium. Figure 4 shows that the oscillation amplitudes increase with increasing initial deviation from geostrophy. The dependence of the oscillation period on the initial squared current Rossby number for different initial deviations from geostrophy in the frame of the fully nonlinear theory described above and in the frame of the weakly nonlinear theory is depicted in Fig. 5. Note that, for the selected range of $m$, the smaller the initial current deviation from geostrophy is, the larger is the deviation of the oscillation period from the inertial period.

\section{b. Oscillation and mean current decay}

In the linear case, the equations in (6) take the form 


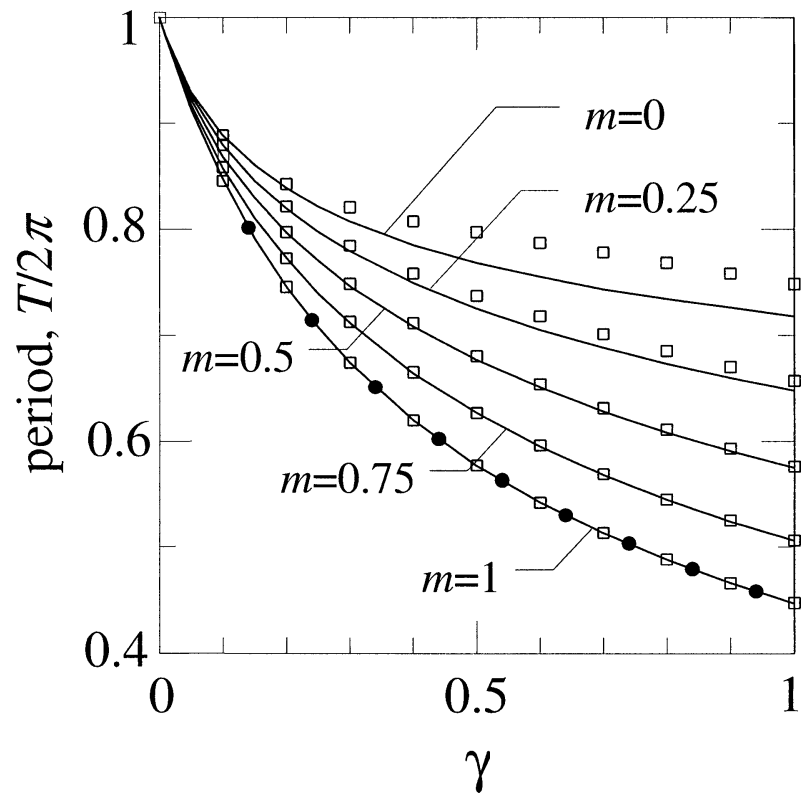

FIG. 5. Period of current oscillations $T$ as a function of the initial current squared Rossby number $\gamma$ for different current deviations from geostrophy (solid lines). The values of the parameter $m$ vary from 0 to 1 , which corresponds to consideration of currents whose initial structures range from quiescence $(m=0)$ to geostrophy $(m=$ 1). The black dots refer to the linear solution (16); the squares refer to the second-order weakly nonlinear solution (25).

$$
\begin{aligned}
\dot{A} & =-s A+B-2 C, & \dot{B} & =-A-s B, \\
\dot{C} & =0, \text { and } & \dot{D} & =0,
\end{aligned}
$$

with

$$
\begin{aligned}
& A(0)=A_{0}, \quad B(0)=B_{0}, \quad C(0)=C_{0}, \quad \text { and } \\
& D(0)=D_{0} .
\end{aligned}
$$

The solution of this problem is

$$
\begin{array}{rlrl}
A & =a_{0} e^{-s t} \sin \Phi-s p_{0}, & B & =a_{0} e^{-s t} \cos \Phi+p_{0}, \\
C & \equiv C_{0}, \quad \text { and } & D \equiv D_{0},
\end{array}
$$

where $\Phi=t+\varphi_{0}, p_{0}=2\left(1+s^{2}\right)^{-1} C_{0}$, and $a_{0}$ and $\varphi_{0}$ are constants that depend on $A_{0}$ and $B_{0}$. If we assume that, in the nonlinear case, amplitude and phase of the oscillations are almost constant during an inertial period, we can conjecture that, also in that case, a solution formally similar to (26) but characterized by time-dependent coefficients $a_{0}, \varphi_{0}$, and $C_{0}$ held. We thus replace the functions $A$ and $B$ in (6) by

$$
\left\{\begin{array}{l}
A \\
B
\end{array}\right\}=a(t) e^{-s t}\left\{\begin{array}{c}
\sin \Phi(t) \\
\cos \Phi(t)
\end{array}\right\}+\left\{\begin{array}{c}
-s \\
1
\end{array}\right\} p
$$

where

$$
p=\frac{2 C(t)}{1+s^{2}},
$$

with $\Phi=t+\varphi(t)$, where now $a, \varphi$, and $C$ are functions of time. Substitution of (27) into (6) yields the following system of ODEs in the unknown time-dependent variables $a, \varphi, C$, and $D$ :

$$
\begin{gathered}
\dot{a}+(*) \sin \Phi+(*) \cos \Phi-\left(^{*}\right) \sin 2 \Phi \\
-(*) \cos 2 \Phi=0, \\
\dot{\varphi}+a p-(*) \sin \Phi-(*) \cos \Phi+(*) \sin 2 \Phi \\
-(*) \cos 2 \Phi=0, \\
\dot{C}-3 \operatorname{sp} C+(*) \sin \Phi=0, \text { and } \\
\dot{D}-s p D+(*) \sin \Phi=0,
\end{gathered}
$$

with $a(0)=a_{0}, \varphi(0)=\varphi_{0}, C(0)=C_{0}$, and $D(0)=$ $D_{0}$. Here (*) are slowly varying functions of time. To find an approximate solution of (28) under the assumptions mentioned above, we apply the method of averaging (e.g., Nayfen 1981) - that is, we average all differential equations of (28) over the temporal interval [0, $2 \pi$ ]. Thus all oscillating terms vanish:

$$
\begin{aligned}
\dot{a} & =0, & \dot{\varphi} & =-2\left(1+s^{2}\right)^{-1} C, \\
\dot{C} & =6 s\left(1+s^{2}\right)^{-1} C^{2}, & \dot{D} & =2 s\left(1+s^{2}\right)^{-1} C D, \\
a(0) & =a_{0}, \quad \varphi(0)=\varphi_{0}, & C(0) & =C_{0}, \quad \text { and } \\
D(0) & =D_{0} . & &
\end{aligned}
$$

The solution of (29) reads

$$
\begin{aligned}
& a=a_{0}, \quad \varphi=\varphi_{0}+(3 s)^{-1} \ln \left(1-3 s p_{0} t\right), \\
& C=C_{0}\left(1-3 s p_{0} t\right)^{-1}, \quad \text { and } \\
& D=D_{0}\left(1-3 s p_{0} t\right)^{-1 / 3} .
\end{aligned}
$$

The expressions (5), (27), and (29) describe the transition of a surface frontal coastal current from an initial stage characterized by intensive superinertial oscillations toward a nonoscillating stage characterized by a decaying mean current. While, as time elapses, the superinertial oscillations decay exponentially the mean current decays potentially, that is, essentially more slowly than the superinertial oscillations. Similar estimates may be also found by replacing the first two equations of (1) with mere geostrophy. This approach was proposed by Matsuura (1980), who investigated numerically the decay of circular frontal vortices. In Fig. 6, an example of frictional decay of different parameters of a surface frontal coastal current is depicted. The fast attenuation of the near-inertial oscillations found in this section accounts for a rapid decay of near-surface oscillatory energy.

\section{Intrinsic current oscillations versus propagating waves}

As we stressed in our introduction and also demonstrated in the last sections, the same coherent mesoscale frontal features involved in the transformation of the 


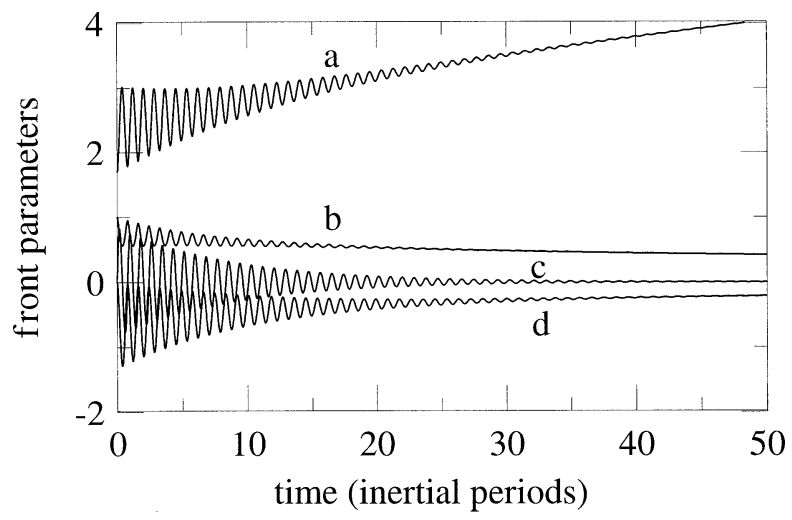

FIG. 6. Frictional decay of a surface frontal coastal current. The time series shows the evolution, according to (6), of (a) the position of the outcropping line, (b) the maximum layer thickness, (c) the cross-front velocity, and (d) the alongfront velocity. The value used for the linear interfacial friction is $s=0.02$. The initial state is rest: $A_{0}=B_{0}=0, C_{0}=-2 \gamma$, and $D_{0}=1$, with $\gamma=0.347$.

spatial scales of oceanic near-inertial internal waves possess intrinsic modes of near-inertial oscillations. Kunze (1985) found that inertial waves interacting with largerscale, anticyclonic geostrophic mesoscale features experience a decrease in their frequency, that is, they become subinertial. One can ask whether our results are in contradiction to the results of Kunze (1985). To answer this question, in this section we analyze smallamplitude disturbances superimposed on a surface frontal current in geostrophic equilibrium:

$u=0, \quad v=V(x)=d H / d x, \quad$ and $\quad h=H(x)$

within the strip $x_{1}<x<x_{2}$, where $H$ is the thickness of the surface frontal layer [with $H\left(x_{1,2}\right)=0$ ] and $V$ is the alongfront, geostrophic velocity. We impose small perturbations $u_{1}, v_{1}$, and $h_{1}$ on (31):

$$
\begin{aligned}
& u=u_{1}(x, t), \quad v=v_{1}(x, t)+V(x), \quad \text { and } \\
& h=h_{1}(x, t)+H(x) .
\end{aligned}
$$

From (1), we obtain the following system of linearized equations:

$$
\begin{aligned}
\frac{\partial u_{1}}{\partial t}-v_{1} & =-\frac{\partial h_{1}}{\partial x}, \\
\frac{\partial v_{1}}{\partial t}+\left(1+\frac{d V}{d x}\right) u_{1} & =0, \quad \text { and } \\
\frac{\partial h_{1}}{\partial t}+V u_{1}+H \frac{\partial u_{1}}{\partial x} & =0 .
\end{aligned}
$$

We now assume that the perturbed fields have the form $h_{1}=Z(x) e^{-i \omega t}, u_{1}=U_{1}(x) e^{-i \omega t}$, and $v_{1}=V_{1}(x) e^{-i \omega t}$. Substituting these expressions in (32) and (33) leads to the boundary-value problem for the unknown variable $Z$ :

$$
\begin{aligned}
\frac{d}{d x}\left[\frac{H}{\omega^{2}-1-d V(x) / d x} \frac{d Z}{d x}\right]+Z & =0, \\
x_{1} & \leq x \leq x_{2}, \quad \text { and } \\
\left|Z\left(x_{1,2}\right)\right| & <\infty,
\end{aligned}
$$

where $\omega^{2}$ is the eigenvalue to be found. The boundary conditions (35) are required to ensure that the solution is bounded at the outcropping lines $x=x_{1,2}$, which are singular points for (34). Note that, in our linear assumption, these boundaries have fixed positions. For surface frontal currents with parabolic cross sections in geostrophic equilibrium we obtain $H=1-\gamma x^{2}, V=$ $-2 \gamma x, x_{1}=-x_{2}$, and $x_{2}=\gamma^{-1 / 2}$. A coordinate transformation according to $x=\xi \gamma^{-1 / 2}$ allows rewriting of (34) and (35) as

$$
\begin{gathered}
\frac{d}{d \xi}\left[\left(1-\xi^{2}\right) \frac{d Z}{d \xi}\right]+\lambda Z=0, \quad-1 \leq \xi \leq 1, \quad \text { and } \\
|Z( \pm 1)|<\infty,
\end{gathered}
$$

where $\lambda=\left(\omega^{2}-1+2 \gamma\right) / \gamma$. Nontrivial solutions of (36) can be expressed in terms of Legendre polynomials for $\lambda=n(n+1), n=0,1, \ldots$ (Abramowitz and Stegun 1972). Hence, for $n>0$, all cross-front disturbances are standing waves of the form

$$
\begin{aligned}
h_{1} & =c_{n} \frac{d^{n}}{d x^{n}}\left[\left(1-\gamma x^{2}\right)^{n}\right] \exp \left(-i \omega_{n} t\right) \quad \text { and } \\
\omega_{n} & =\sqrt{1+[n(n+1)-2] \gamma}
\end{aligned}
$$

where $c_{n}$ are arbitrary constants and $|x| \leq \gamma^{-1 / 2}$. The form of the frontal interface is asymmetric (symmetric) with respect to the current axis, if $n$ is odd (even). The frequency $\omega_{n}$ is exactly inertial for $n=1$ and superinertial for $n>1$. Note that, in the case of surface frontal coastal currents, only symmetric modes (even $n$ ) are allowed. For the lowest symmetric mode of oscillations $(n=2)$, the disturbance is parabolic and the relations (16) and (37) coincide. For $n=0$ we obtain

$h_{1}=\mathrm{const} \quad$ and $\omega_{0}=\sqrt{1-2 \gamma} \approx 1+\frac{1}{2} \frac{d V}{d x}$

for $0<\gamma<0.5$. The frequency of the disturbance is thus subinertial and corresponds to the approximate dispersion relation derived by Kunze (1985). The solution (38) simply results from (32), for vanishing gradient of the perturbed thickness, but cannot fulfill the continuity equation in (33), and hence it cannot be considered as a simple mode of oscillation of the surface frontal current. The solution presented here for $n=0$ and that presented by Kunze (1985) can be seen as a superposition of inertial oscillations on a background vorticity field. 


\section{Discussion and conclusions}

In this paper we have studied, in the frame of the nonlinear reduced-gravity theory, intrinsic oscillations of stable surface frontal currents of special structure. In particular, we have concentrated our attention on their inviscid evolution and on their frictional decay. The motivation for our investigation is the need of gaining a deeper understanding of fundamental aspects related to the near-inertial evolution of these oceanic mesoscale features, as a prerequisite toward a better comprehension of the physical mechanisms involved in the transformation of longer, mostly wind-induced near-surface inertial oscillations in shorter near-inertial oscillations that are able to transfer their energy rapidly to the oceanic abyss. In fact, the inhomogeneities in the oceanic vorticity field that, on an $f$ plane, are responsible for this scale transformation often coincide with near-surface mesoscale frontal features, which are known to possess intrinsic modes of near-inertial oscillations.

In the case of double-fronted surface currents characterized by parabolic cross sections and linear horizontal velocity structure we were able to reduce the nonlinear reduced-gravity shallow-water equations to a set of nonlinear, coupled ordinary differential equations. In the inviscid case, a periodic analytical solution of the nonlinear problem describing the inertially reversing horizontal displacement of a surface frontal current having a fixed parabolic cross section was found. The inviscid cross-front pulsation of a coastal current emerging from a motionless surface frontal layer was also investigated analytically. In this case, it was found that, for any current size and oscillation amplitude, the oscillations are superinertial. In the linear and in the weakly nonlinear approximation, different aspects of the pulsating dynamics of surface frontal currents were investigated. For infinitesimal disturbances imposed on a geostrophic current, a simple formula relates oscillation frequency and squared current Rossby number: $\omega=$ $\sqrt{1+4 \gamma}$. Whereas disturbances of the geostrophic current imposed in the alongfront direction result in oscillation periods shorter or larger than the linear one, disturbances in the cross-front direction yield always shorter periods than in the linear case. This result is a consequence of the fact that alongfront velocity disturbances not only generate oscillations but also modify the current Rossby number. On the contrary, crossfront velocity disturbances are not able to change substantially the current Rossby number. The effect of linear interface friction was also investigated: the current oscillations decay according to an exponential law, whereas the intensity of the mean current decays according to a power law. Note also that instability mechanisms (which have not been considered in this investigation) can contribute to the energy decay of frontal mesoscale features (Griffiths et al. 1982; Paldor and Killworth 1987; Rubino et al. 2002). In a linear context, it was found that disturbances superimposed on the sur- face frontal current are standing waves within the bounded region, whose frequencies are inertial/superinertial for the first mode/higher modes. In the same frame, a zeroth mode, referring to the superposition of an inertial wave on a background vorticity field and hence resembling the situation investigated by Kunze (1985), would formally yield subinertial frequencies. Note that the approach used in our investigation-that is, the reduced-gravity assumption-does not allow one to study vertical propagation of near-inertial activity from the upper to the lower ocean. Such propagation would crucially depend on the properties of an active ambient ocean such as, for example, density and velocity distribution (Kunze 1985; Wang 1991; Young and Jelloul 1997; Balmforth et al. 1998). Although we recognize that an extension of our analytical work to include an active ambient ocean may be unfeasible, multilayer numerical models could be used to quantify this vertical energy transfer (e.g., Wang 1991).

Still, a deeper comprehension of the intrinsic oscillations of geophysical frontal features in a simplified context may represent a prerequisite for better understanding the intricate dynamics leading to the observed near-inertial wave field in the ocean. In fact, in the upper ocean, the energy of the wind excites inertial waves having similar spatial scales as the generating atmospheric fronts. Such waves need to experience a variation in their frequency to be able to transfer their energy rapidly downward. Together with their equatorward propagation (Garrett 2001), interactions of inertial waves with inhomogeneities of the ambient vorticity field (Kunze 1985) are able to produce such a frequency shift. On the other hand, however, and mostly depending on their specific geometry, many of the same inhomogeneities possess intrinsic modes of near-inertial oscillations, which would possibly generate near-inertial waves in an active ambient ocean. Thus, on an $f$ plane, the zoo of existing surface frontal mesoscale features may play a larger role than believed in the past in the observed rapid propagation of the energy of the wind toward the abyss.

Acknowledgments. This study has been partly funded by the Deutsche Forschungsgemeinschaft in the frame of the Sonderforschungsbereich 512 and by the Deutscher Akademischer Austauschdienst (DAAD).

\section{REFERENCES}

Abramowitz, M., and L. A. Stegun, 1972: Handbook of Mathematical Functions with Formulas, Graphs, and Mathematical Tables. U. S. Government Printing Office, 1046 pp.

Ball, F. K., 1963: Some general theorems concerning the finite motion of a shallow rotating liquid lying on a paraboloid. J. Fluid Mech., 17, 240-256.

Balmforth, N. J., S. G. Llwellyn Smith, and W. R. Young, 1998: Enhanced dispersion of near-inertial waves in an idealized geostrophic flow. J. Mar. Res., 56, 1-40.

Chant, R., 2001: Evolution of near-inertial waves during an upwelling 
event on the New Jersey inner shelf. J. Phys. Oceanogr., 31, 746-764.

Cushman-Roisin, B., 1987: Exact analytical solutions for elliptical warm-core rings. Tellus, 39A, 235-244.

- W. H. Heil, and D. Nof, 1985: Oscillations and rotations of elliptical warm-core rings. J. Geophys. Res., 90, 11 756-11 764.

Frei, C., 1993: Dynamics of a two-dimensional ribbon of shallow water on an $f$-plane. Tellus, 45A, 44-53.

Garrett, C., 2001: What is the "near-inertial" band and why is it different from the rest of the internal wave spectrum? J. Phys. Oceanogr., 31, 962-971.

Gradshteyn, I. S., and I. M. Ryzhik, 1980: Table of Integrals, Series, and Products. Academic Press, $1160 \mathrm{pp}$.

Griffiths, R. W., P. D. Killworth, and M. E. Stern, 1982: Ageostrophic instability of ocean currents. J. Fluid Mech., 117, 343-377.

Klein, P., and A. M. Treguier, 1993: Inertial resonance induced by an oceanic jet. J. Phys. Oceanogr., 23, 1897-1915.

Kunze, E., 1985: Near-inertial wave propagation in geostrophic shear. J. Phys. Oceanogr., 15, 544-565.

_ 1986: The mean and near-inertial velocity fields in a warmcore ring. J. Phys. Oceanogr., 16, 1444-1461.

- and T. B. Sanford, 1984: Observations of near-inertial waves in a front. J. Phys. Oceanogr., 14, 566-581.

Lee, C. M., and C. C. Eriksen, 1997: Near inertial internal wave interactions with mesoscale fronts: Observations and models. $J$. Geophys. Res., 102, 3237-3253.

Matsuura, T., 1980: On a decay process of isolated, intense vortexes in a two-layer ocean. J. Oceanogr. Soc. Japan, 36, 39-45.

Mied, R. P., C. Y. Shen, C. L. Trump, and G. J. Lindermann, 1986: Inertial-internal waves in a Sargasso Sea front. J. Phys. Oceanogr., 16, 1751-1762.

Mooers, C. N. K., 1975: Several effects of a baroclinic current on the cross-stream propagation of inertial-internal waves. Geophys. Fluid Dyn., 6, 245-275.
Munk, W. H., 1981: Internal waves and small-scale processes. Evolution of Physical Oceanography, B. A. Warren and C. Wunsch, Eds., The MIT Press, 264-291.

Nayfen, A. H., 1981: Introduction to Perturbation Techniques. John Wiley and Sons, $519 \mathrm{pp}$.

Paldor, N., and P. D. Killworth, 1987: Instabilities of a two-layer coupled front. Deep-Sea Res., 34, 1525-1539.

Rogers, C., 1989: Elliptic warm-core theory: The pulsrodon. Phys. Lett., 138, 267-273.

Rubenstein, D. M., and G. O. Roberts, 1986: Scattering of inertial waves by an ocean front. J. Phys. Oceanogr., 16, 121-131.

Rubino, A., and P. Brandt, 2003: Warm-core eddies studied by laboratory experiments and numerical modeling. J. Phys. Oceanogr., 33, 431-435.

, -, and K. Hessner, 1998: Analytical solutions for circular eddies of the reduced-gravity, shallow-water equations. J. Phys. Oceanogr., 28, 999-1002.

—, K. Hessner, and P. Brandt, 2002: The decay of stable frontal warm-core eddies in a layered frontal model. J. Phys. Oceanogr., 32, $188-201$.

Shapiro, A., 1996: Nonlinear shallow-water oscillations in a parabolic channel: Exact solutions and trajectory analyses. J. Fluid Mech., 318, 49-76.

van Meurs, P., 1998: Interaction between near-inertial mixed layer currents and the mesoscale: The importance of spatial variabilities in the vorticity field. J. Phys. Oceanogr., 28, 1363-1388.

Wang, D.-P., 1991: Generation and propagation of inertial waves in the subtropical front. J. Mar. Res., 49, 619-633.

Young, W. R., 1986: Elliptical vortices in shallow water. J. Fluid Mech., 171, 101-119.

—_, and M. B. Jelloul, 1997: Propagation of near-inertial oscillations through a geostrophic flow. J. Mar. Res., 55, 735-766.

Zervakis, V., and M. D. Levine, 1995: Near-inertial energy propagation from the mixed layer: Theoretical considerations. J. Phys. Oceanogr., 25, 2872-2889. 